Sitientibus Série Ciências Físicas 03: 10-17 (2007)

\title{
O Letramento em Ciências como Objetivo para o Ensino de Ciências*
}

\author{
The Science Literacy as an Objective for the Science Teaching
}

\author{
Katemari Rosa ${ }^{\dagger}$ \\ Departamento de Física - UEFS \\ Av. Transnordestina, s/n, Km 03, BR 116 \\ Feira de Santana - BA - 44031-460 \\ Maria Cristina Martins $\ddagger$ \\ Instituto de Física - UFBA \\ Campus Universitário de Ondina \\ Salvador - BA - 40210-340
}

\begin{abstract}
Neste artigo apresentamos uma discussão sobre o significado de alfabetização científica e suas implicações para o ensino de ciências. Inicialmente discutimos a relação entre alfabetização e letramento e em seguida mostramos que o termo "alfabetização científica" abarca diferentes conceitos presentes na literatura através dos tempos. A importância dessa discussão emerge num ano em que o Brasil foi novamente submetido a uma avaliação comparativa de estudantes no que se refere à "alfabetização científica", o PISA (Programme for International Student Assessment). Essa avaliação, que teve início no ano de 2000, tem mostrado que somos um país que pouco sabe sobre ciência, pois o país logrou as últimas colocações nas avaliações de anos anteriores. A alfabetização científica vem se mostrando como um objetivo no ensino de ciências no Brasil e no mundo. Nesse sentido é importante que se discuta o que é alfabetização científica, para melhor entendermos e analisarmos as avaliações a que somos submetidos. Mais do que isso, para que possamos bem avaliar nosso trabalho, assim como planejar nossas ações didáticas.
\end{abstract}

\section{Palavras-chaves: Ensino de Ciências, Alfabetização Científica, PISA.}

In this work we show a discussion about the significance of science literacy and its implications for science teaching. Firstly we discuss a relation between two forms of literacy and following we show that the term "science literacy" encompasses different concepts existing in the literature over the times. The relevance of this discuss arises at a year that Brazil was again submitted to a comparative evaluation of students related to "science literacy", the PISA (Programme for International Student Assessment). This evaluation, whose beginning was in the year of 2000, has shown we are a country that known little about science, since the country has achieved the last places in classification on previous years. The science literacy has become a goal for teaching science on Brazil and around the world. In this sense, it is important to discuss what it is science literacy in order to understand and analyze the evaluations which we are submitted. More than that, to able us to better evaluate well our work as well as plan our didactic actions.

Key-words: Science Teaching, Science Literacy, PISA

\section{INTRODUÇÃO}

\footnotetext{
* Texto adaptado e revisado, originalmente apresentado no XVII Simpósio Nacional para o Ensino de Física, com o título original "O que é Alfabetização Científica, afinal?

${ }^{\dagger}$ Endereço Eletrônico: katemari@gmail.com

${ }^{\ddagger}$ Endereço Eletrônico: mcristi@ufba.br
}

À expressão "alfabetização científica" (science literacy) tem sido atribuídos diferentes significados e papéis (Matthews (1994) [1]; Auler \& Delizoicov (2001) [2]; Lorenzetti \& 
Delizoicov (2001) [3]; KEMP (2000 e 2002) [4, 5]; Chassot (2003) [6]; Díaz et al. (2003) [7]. Aqui pretendemos refletir sobre algumas destas conceituações e qual entendemos ser o papel de uma alfabetização científica.

\section{LETRAMENTO EM CIENCIAS}

O termo science literacy teria uma tradução mais fiel como "letramento em ciências" ao invés de "alfabetização científica", ainda que, na língua portuguesa, alfabetização seja uma acepção possível, com a idéia de processo. Entretanto a própria palavra (letramento) surge na língua portuguesa na década de 80 por influência do inglês (literacy) (Houaiss (2001) [8]). Outrossim, "letramento" dá muito mais a dimensão de como entendemos a "alfabetização científica", no sentido de cultura mais ampla, de possibilidade de transitar numa determinada área, discutir sobre seus problemas ou, mais modesta e acertadamente, entender discussões travadas sobre seus problemas. Portanto vemos "alfabetização científica" como uma iniciação, uma inserção na cultura científica. Contudo, essa iniciação tem sido um problema enfrentado mundialmente pela comunidade de educadores. Problema esse identificado como a "crise da alfabetização científica" que, segundo Matthews (1994) [1] surgiu na década de 80 .

$\mathrm{O}$ autor mostra o panorama dessa crise nos Estados Unidos ao afirmar que, nesse período, o fato era que os estudantes estadunidenses não faziam muitos cursos na área científica, que eles não sabiam muito sobre ciências e importavamse pouco sobre aprender ciências. Além disso, o Matthews (1994) [1] alega que uma pesquisa monitorando o conhecimento científico de adolescentes, desde 1969, mostrava que os escores para todas as faixas etárias estudadas diminuíram nos anos 70, melhoraram um pouco nos anos 80, mas nos anos 90 foram os mesmos dos 70, dentro de um grupo, e menor ainda, em outro. Ou seja, que o conhecimento científico dos adolescentes estadunidenses vem diminuindo.
"Uma nação em risco" foi uma publicação que tornou o assunto da crise educacional popular e pressionou o governo dos Estados Unidos a tomar algumas atitudes, e seus autores, de certa forma, definiram alfabetização científica como:

The teaching of science in high school should provide graduates with an introduction to: (a) the concepts, laws, and processes of the physical and biological sciences; (b) the methods of scientific inquiry and reasoning; (c) the applications of scientific knowledge to everyday life, and (d) the social and environmental implications of scientific and technological development. (National Commission on Excellence in Education, apud Matthews, p. 31, [1])[13]

Matthews salienta que há várias formas de definir alfabetização científica, desde uma definição restrita onde a alfabetização é a capacidade de reconhecer fórmulas e dar definições corretas, até uma definição mais expansiva que inclui o entendimento dos conceitos e algum grau de compreensão sobre a natureza da ciência e suas dimensões sociais e históricas. O autor defende uma definição mais expansiva, e reconhece que essa definição exige do professor, como muitos outros problemas que esses enfrentam, uma visão de "boa educação", filosofia da educação. Para Paul Dehart Hurd (Matthews (1994) [1]),

A measure of scientific literacy is a measure of cultural awareness. The traditional science curriculum leaves students foreigners in their own culture. A problem in bringing about the essential reform of science teaching is that there are too many scientists who are scientifically illiterate and too few philosophers, sociologists, and historians of science and technology who are interested in precollege science education (Hurd 
(1958) apud Matthews, IBID, p. 32 [1]).[14]

A National Science Teachers Association, em 1982, definia uma pessoa letrada cientificamente aquela que entendia que a sociedade controlava a ciência e a tecnologia através da alocação de recursos; que usava os conceitos, habilidades e valores científicos para tomar decisões cotidianas; que reconhece tanto as limitações quanto as utilidades da ciência e tecnologia para o progresso da riqueza da humanidade; que sabe os principais conceitos, hipóteses e teorias da ciência e está apto a usálos; que distingue entre evidências científicas e opiniões pessoais; que tem uma visão mais rica do mundo como um resultado da educação científica; e que conhece fontes confiáveis de informações tecnológicas e científicas e as utiliza em processos de tomada de decisão (NSTA (1982) apud Matthews (1994), p. 32 [1]).

Auler \& Delizoicov (2001) [2] utilizam a expressão "alfabetização científico-tecnológica" (ACT) e argumentam que essa abarca um amplo espectro de significados, que podem ser traduzidos através de expressões como popularização da ciência, divulgação científica, entendimento público da ciência e democratização da ciência. Ainda destacam que a ACT tem tido, basicamente, dois encaminhamentos: a perspectiva reducionista que entendem como um simples incremento do atual ensino de Ciências, desconsiderando a existência de construções subjacentes à produção do conhecimento Científico-Tecnológico, tendo uma concepção de neutralidade da ciência e tecnologia (CT); e a perspectiva ampliada como uma busca da compreensão de interações entre Ciência-Tecnologia-Sociedade (CTS), associando o ensino de conceitos à problematização de construções históricas vinculadas à suposta neutralidade da CT.

Lorenzetti \& Delizoicov (2001) [3] partem das conceituações de alfabetização, na aquisição da língua, do processo de representação de fonemas em grafemas, e do processo de compreensão e expressão de significados através do código escrito, para discutirem a alfabetização científica nas séries iniciais.
Buscam em Leite \& Souza uma definição mais ampla "o processo de alfabetização deve, desde o início, possibilitar que o aluno desenvolva a compreensão do caráter simbólico da escrita (instrumento que serve para transmitir idéias, emoções, impressões etc.), além de identificar todas as possibilidades sociais de seu uso" (Leite \& Souza (1995), p. 16 apud Lorenzetti \& Delizoicov (2001), p. 8 [3])[15] e afirmam que essa está próxima de um conceito de letramento.

Pode-se dizer que o letramento
$e ́$ o uso que as pessoas fazem
da leitura e da escrita em seu
contexto social. Convivendo com
uma variedade muito grande de in-
formaçôes, almeja-se que as pes-
soas saibam compreender os sig-
nificados que os textos propiciam,
incorporando-os na sua prática so-
cial. O indivíduo poderá fazer uso
competente e frequente da leitura
e da escrita em seu trabalho, em
casa, no seu lazer, etc. (Lorenzetti
$\&$ Delizoicov (2001), p. 8 [3])

Nesse sentido nossa assumpção[16] de que letramento seria um termo mais adequado encontra coro nas palavras de Lorenzetti \& Delizoicov [3], pois entendemos que "alfabetização científica" passa, sim, pelo reconhecimento da linguagem, dos símbolos, dos códigos da ciência, mas vai além, e só faz sentido quando o indivíduo é capaz de incorporar e utilizar esse conhecimento socialmente, ou seja apropria-se dele, acrescentando-o a sua cultura.

O conceito de letramento é também utilizado no atual programa internacional de avaliação comparada, o PISA, que tem a finalidade de produzir indicadores sobre a efetividade dos sistemas educacionais, avaliando o desempenho de alunos na faixa dos 15 anos, idade em que se pressupõe o término da escolaridade básica obrigatória na maioria dos países (INEP (2006) [9]). O PISA define letramento em ciências como:

Envolve o uso de conceitos científicos necessários para compreender e ajudar a tomar decisões 
sobre o mundo natural. Também envolve a capacidade de reconhecer questões científicas, fazer uso de evidências, tirar conclusões com bases científicas e comunicar essas conclusões. São utilizados conceitos científicos que serão relevantes para serem usados pelos alunos tanto no presente quanto no futuro próximo. (INEP (2006) [9]).

E é dentro desse conceito de letramento que o Brasil ficou em último lugar na avaliação de 2000 e em penúltimo lugar em 2004, com o Peru em último lugar. A avaliação de 2000 contou com 37 países participantes e a ênfase das provas era na leitura, em 2004 foram 41 países e a ênfase foi para a matemática; em 2006 ocorreu nova avaliação, com ênfase em ciências, mas os resultados ainda não foram divulgados.

Chassot (2003) [6] também critica o termo "alfabetização", entretanto afirma que "letrado" apresenta "conotações pernósticas" e aponta que em Portugal utiliza-se a palavra "literacia". Etimologias à parte, o autor considera "alfabetização científica" como um "conjunto de conhecimentos que facilitariam aos homens e mulheres fazer uma leitura do mundo onde vivem". Também se apóia na crítica feita em relação à língua materna, quando se exige dos alfabetizados mais do que ler e escrever, cobrando criticidade. Nesse sentido, diz que é desejável que "os alfabetizados cientificamente não apenas tivessem facilitada a leitura do mundo em que vivem, mas entendessem as necessidades de transformá-lo, e transformá-lo para melhor" (grifo do autor) (IBID, p. 38 [6]).

Identificamos alfabetização científica como parte de uma agenda política ao encontrarmos em Díaz et al. (2003) [7] que essas diferentes definições estão refletidas explicitamente nos informes de política educativa de órgãos como a UNESCO, o International Council for Science [17], o International Bureau of Education [18] e a Organização dos Estados Iberoamericanos para a Educação, Ciência e Cultura (OEI), além de estarem presentes nos objetivos de vários projetos de ensino como o Pro- jeto 2061 - Ciência para Todos - da American Association for the Advancement of Science [19] (AAAS), ou associações profissionais com grande influência em projetos de ensino: a International Technology Education Association [20] (ITEA), a National Science Teachers Association [21] (NSTA) e o National Research Council [22] (NRC).

A alfabetização científica parece estar na "moda", e precisamos ter um olhar crítico quando pensamos nela como um objetivo para o ensino de ciências. É particularmente interessante um dos sentidos que Díaz et al. apresentam sobre o tema, pois traz a idéia de que a alfabetização científica não é um objetivo possível, mas desejável:

\section{Mito cultural (Shamos (1995))}

que, aunque expresado originalmente desde una perspectiva crítica, se puede reformular como la utopía que señala el ideal a perseguir . (Diaz et al., p. 3 (2003)).[23]

Assim, para além de "mito cultural", a alfabetização científica seria um "lema" que representaria os propósitos das reformas no ensino de ciências e de um movimento internacional de especialistas em educação científica. Esse "lema", entretanto, sofre críticas e uma delas é: ao tempo em que existem várias definições, conceitos, objetivos para a "alfabetização científica", nenhum consegue expressar uma idéia unívoca, porque a gama que o termo abarca é suficientemente grande para abrigar contradições. De acordo com Kemp (2002), Shamos (1995) argumenta que falta legitimidade nos pedidos de apoio aos objetivos da alfabetização científica, e diz que há pouca ou nenhuma evidência de que uma alfabetização científica seja necessária para que as pessoas atinjam o sucesso ou tenham uma vida boa. Kemp traz também as críticas de Atkin \& Helms (1993) e Laugksch (2000)[24] que vão no sentido de que, para o primeiro, as razões para a alfabetização científica vêm se acumulando, mas não há um exame crítico para ver se são todas compatíveis umas com as outras ou até desejáveis; e no segundo, de que mesmo no séc. 
XXI há uma visão de alfabetização científica má definida, tratando-se de um conceito difuso.

Para Shamos, "there is no consensus on what 'scientific literacy' means or should mean. Instead, everyone involved with science education appears to have a vague, ill-defined notion of what it should mean." [25] (Shamos p. 160, (1995) apud Kemp, p. 1 (2002)). Entendemos a dificuldade que essa maleabilidade do conceito traz para a pesquisa em ensino de ciências, mas acreditamos que é possível trabalhar com os consensos que existem. Nesse sentido, ao analisar a percepção sobre alfabetização científica de nove especialistas em Didática das Ciências, Kemp (2002) [5] identificou pontos em comum e classificou as idéias em três dimensões que compreenderiam o conceito de "alfabetização científica": conceitual, procedimental e afetiva:

Conceitual: envolve a compreensão e conhecimentos específicos. Termos que a identificam: conceitos de ciência e relações entre ciência e sociedade;

Procedimental: envolve os procedimentos, processos, habilidades e capacidades. Termos relacionados: obtenção e uso da informação científica, aplicação da ciência na vida cotidiana, utilização da ciência para fins sociais e cívicos, e divulgação científica ao público de maneira acessível;

Afetiva: envolve emoções, atitudes, valores e disposição para a alfabetização científica. Elementos: gostar de ciência, interesse por ciência.

\section{BUSCANDO CONSENSOS}

Nosso trabalho tem "objetivos modestos" [26] (Matthews (1998) [11]) e partindo de diferentes visões de alfabetização científica, das dimensões identificadas por Kemp, concentra-se ainda mais. Numa revisão sobre a concepção de educadores em ciências dos objetivos de uma alfabetização científica,
Kemp (2000) [4] analisa trabalhos de 1952 a 1998 da literatura estadunidense, e produz tabelas onde lista os diferentes elementos para uma alfabetização científica que encontrou em sua pesquisa. Ele organiza três tabelas: na primeira traz os elementos encontrados de 1952 até 1963, na segunda, compara todos elementos já encontrados com o que vê de 1964 até 1982, e na terceira, faz mais uma compilação (de 1952 até 1982) e compara com o que se apresenta de 1982 até 1998. Desses dados produzimos a tabela 1 . Nela colocamos os autores dos trabalhos analisados identificados de 1 a 19, assim, por exemplo, o "1" refere-se a um trabalho de Conant publicado em 1952, na provável primeira utilização do termo science literacy.

Além dos elementos da alfabetização científica, podemos observar nesta tabela quais os elementos que aparecem mais consensualmente nos artigos analisados (KEMP (2000) [4]):

Independência intelectual - que inclui ter habilidade de procurar informaçoes e tomar decisões próprias acerca de questões envolvendo ciência, bem como ter a habilidade de avaliar conselhos de especialistas. Além disso, ser capaz de aprender ciências após o período de educação formal.

Comunicação em ciências - refere-se às habilidades necessárias tanto para interpretar comunicações científicas, através da mídia escrita, em áudio, visual, quanto para realizar este tipo de comunicação, ou seja, ser capaz de escrever, falar, sobre assuntos que envolvam ciência;

Conhecimento conceitual - que se refere ao conhecimento de várias disciplinias científicas, as menções consideradas nesse elemento envolvem uma gama de disciplinas científicas, não restritas a uma ou duas;

Natureza da ciência - compreendendo conhecimentos sobre hipóteses, evidências, o caráter tentativo da ciência, a falibilidade do empreendimento científico, com sua constante auto correção.

Apesar das críticas apresentadas, consideramos que esses consensos identificados na literatura podem ser balizadores para a busca de um letramento em ciências. Nesse sentido, a 
tabela acima nos mostra quatro elementos consensuais e consideramos que esses podem ser tomados, modestamente, como objetivos para o letramento em ciências. Enfatizamos o caráter "modesto", pois percebemos que existem ou- tras componentes desse letramento, mas entendemos ser necessário estabelecer alguns focos e para isso valemo-nos dos consensos já produzidos.

\begin{tabular}{|c|c|c|c|c|c|c|c|c|c|c|c|c|c|c|c|c|c|c|c|}
\hline Autores & & 1952 & $2-1$ & 963 & & & & 1964 & $4-1$ & 982 & & & & & 198: & $3-1$ & 998 & & \\
\hline Elementos & 1 & 2 & 3 & 4 & 5 & 6 & 7 & 8 & 9 & 10 & 11 & 12 & 13 & 14 & 15 & 16 & 17 & 18 & 19 \\
\hline Independência intelectual & $\mathrm{X}$ & & & & & $\mathrm{X}$ & & & $\mathrm{X}$ & & & $\mathrm{X}$ & $\mathrm{X}$ & $\mathrm{X}$ & $\mathrm{X}$ & $\mathrm{X}$ & $\mathrm{X}$ & $\mathrm{X}$ & $\mathrm{X}$ \\
\hline Comunicação em ciências & $\mathrm{X}$ & $\mathrm{X}$ & $\mathrm{X}$ & & $\mathrm{X}$ & $\mathrm{X}$ & & & & & $\mathrm{X}$ & & $\mathrm{X}$ & $\mathrm{X}$ & $\mathrm{X}$ & $*$ & $\mathrm{X}$ & $\mathrm{X}$ & $\mathrm{X}$ \\
\hline Ciência e sociedade & $\mathrm{X}$ & $\mathrm{X}$ & $\mathrm{X}$ & & & $\mathrm{X}$ & $\mathrm{X}$ & $\mathrm{X}$ & $\mathrm{X}$ & $\mathrm{X}$ & $\mathrm{X}$ & $\mathrm{X}$ & $\mathrm{X}$ & $\mathrm{X}$ & & $\mathrm{X}$ & $\mathrm{X}$ & $\mathrm{X}$ & $\mathrm{X}$ \\
\hline Conhecimento conceitual & $\mathrm{X}$ & $\mathrm{X}$ & $\mathrm{X}$ & $\mathrm{X}$ & $\mathrm{X}$ & $\mathrm{X}$ & $\mathrm{X}$ & $\mathrm{X}$ & $\mathrm{X}$ & $\mathrm{X}$ & $\mathrm{X}$ & $\mathrm{X}$ & $\mathrm{X}$ & $\mathrm{X}$ & $\mathrm{X}$ & $\mathrm{X}$ & $\mathrm{X}$ & $\mathrm{X}$ & $\mathrm{X}$ \\
\hline Ciência e tecnologia & $\mathrm{X}$ & & $\mathrm{X}$ & & & & $\mathrm{X}$ & $\mathrm{X}$ & $\mathrm{X}$ & $\mathrm{X}$ & $\mathrm{X}$ & $\mathrm{X}$ & & & & $\mathrm{X}$ & $\mathrm{X}$ & $\mathrm{X}$ & $\mathrm{X}$ \\
\hline Ciência e cotidiano & $\mathrm{X}$ & & & $\mathrm{X}$ & & $\mathrm{X}$ & & & $\mathrm{X}$ & & $\mathrm{X}$ & $\mathrm{X}$ & & $\mathrm{X}$ & $\mathrm{X}$ & $\mathrm{X}$ & $\mathrm{X}$ & $\mathrm{X}$ & $\mathrm{X}$ \\
\hline Ética da ciência & & $\mathrm{X}$ & & & & & & & & $\mathrm{X}$ & & & & $\mathrm{X}$ & & $\mathrm{X}$ & & $\mathrm{X}$ & $\mathrm{X}$ \\
\hline Natureza da ciência & & & $\mathrm{X}$ & & $\mathrm{X}$ & & $\mathrm{X}$ & $\mathrm{X}$ & & $\mathrm{X}$ & & $\mathrm{X}$ & $\mathrm{X}$ & $\mathrm{X}$ & $\mathrm{X}$ & $\mathrm{X}$ & $\mathrm{X}$ & $\mathrm{X}$ & $\mathrm{X}$ \\
\hline História da ciência & & & & $\mathrm{X}$ & & & & & & & & & & $\mathrm{X}$ & & $\mathrm{X}$ & $\mathrm{X}$ & $\mathrm{X}$ & \\
\hline Apreciação da ciência & $\mathrm{X}$ & & $\mathrm{X}$ & $\mathrm{X}$ & & & $\mathrm{X}$ & $\mathrm{X}$ & & & $\mathrm{X}$ & $\mathrm{X}$ & & $\mathrm{X}$ & $\mathrm{X}$ & $\mathrm{X}$ & $\mathrm{X}$ & $\mathrm{X}$ & \\
\hline Ciência nas humanidades & & & & & & & & & $\mathrm{X}$ & $\mathrm{X}$ & & & & $\mathrm{X}$ & & & & & \\
\hline Aptidões cientificas & & & & & & & & & & & & & & & $\mathrm{X}$ & & & $\mathrm{X}$ & \\
\hline Ciência e matemática & & & & & & & & & & & & & & & & $\mathrm{X}$ & $\mathrm{X}$ & $\mathrm{X}$ & \\
\hline
\end{tabular}

Fonte: Andrew C. Kemp, 2000, modificada.

FIGURA 1: Elementos da "Alfabetização Científica" de 1952 - 1998.

\section{ALGUMAS CONSIDERAÇÕES}

A alfabetização científica vem se mostrando como um objetivo no ensino de ciências no Brasil e no mundo. Nesse sentido é importante que se discuta o que é alfabetização científica, para melhor entendermos e analisarmos as avaliações a que somos submetidos. Mais do que isso, para que possamos bem avaliar nosso trabalho.

Quanto à terminologia "alfabetização" ou "letramento", verificamos que muitos autores utilizam o primeiro tanto para fazer referência a uma iniciação, um primeiro contato com as ciências, quanto para compartilhamento de uma cultura científica, tendo uma gama de acepções, inclusive englobando o sentido de letramento; enquanto o segundo termo é usado apenas num sentindo mais amplo, incluindo o conhecimento dos conceitos, da linguagem matemática e do compartilhamento da cultura científica.

A despeito da pluralidade de acepções que encerra, verificamos que há alguns consensos entre os pesquisadores, e consideramos que futuros trabalhos e avaliações podem ser fundamentados a partir desses consensos, o que amadurece a discussão e fortalece nossos esforços para atingir um objetivo comum, o letramento em ciências.

Além disso, esperamos que a revisão aqui discutida contribua para, o planejamento de nossas ações didáticas em prol deste letramento. 
[1] M.R. Matthews, Science Teaching - The role of history and philosophy of science. London: Routledge (1994).

[2] D. Auler, D. Delizoicov, Alfabetização científico-tecnológico para quê?. Ensaio Pesquisa em Educação em Ciências 3, (1) (2001).

[3] L. Lorenzetti, D. Delizoicov, Alfabetização científica nocontexto das séries iniciais. Ensaio - Pesquisa em Educação em Ciências 3, (1) (2001).

[4] C.A. Kemp, Science Educator's views on th Goal of Scientific Literacy for All: An Interpretive Review of the Literature. In: Annual Meeting of Teachers in Science, 2000. New Orleans: (2000). Disponível em: http://www.eric.ed.gov/ERICDocs/data/eric docs2/content_storage_01/0000000b/80/26/ 16/de.pdf. Visualizado em: 07-jan.(2006).

[5] C.A. Kemp, Implcations of diverse meanings for "scientific literacy". In: Proceedings of The Annual International Conference of the Association for the Education of Teachers in Science, 2002. Charlotte: (2002). Disponível em: http://www.ed.psu.edu/CI/Journals/2002aets /s3_kemp.rtf. Visualizado em: 06-jan.(2006).

[6] A. Chassot, Alfabetização científica: questões e desafios para a educação. Ijuí: Editora UNIJUÍ (2000).

[7] J.A.A. Díaz, V. Ángel, M.A.M. Mas, Papel de la educación CTS en una alfabetización científica y tecnológica para todas las personas. Revista Electrónica de Enseñanza de las Ciencias 2, (2) (2003).

[8] A. Houaiss, M.S. Villar, F.M.M. Franzo, Dicionário Houaiss da Língua Portuguesa. Rio de Janeiro: Objetiva (2001).

[9] Instituto Nacional de Estudos e Pesquisas Educacionais Anísio Teixeira (INEP), Alfabetização científicotecnológico para quê?. Disponível em: http://www.inep.gov.br/internacional/pisa/. Visualizado em: 06-jan.(2006).

[10] M.R. Matthews, História, filosofia e ensino de ciências: a tendência atual de reaproximação. Caderno Catarinense de Ensino de Física 12, (3) 164 (1993).

[11] M.R. Matthews, Indefense of modest goals when teaching about the nature of science. Journal of Research in Science Teaching 35, (2) 161 (1998).

[12] The National Science Teachers Associa- tion (NSTA), Press release. Disponível em: http://www.nsta.org/pressroom\&news_story_ $\mathrm{ID}=47015$. Visualizado em: 07-jan. (2006).

[13] "O Ensino de ciências no ensino médio deveria formar estudantes com uma introdução a: (a) conceitos, leis e processos das ciências físicas e biológicas; (b) métodos da racionalidade e da investigação científica; (c) aplicações do conhecimento científico no cotidiano; e (d) implicações sociais e ambientais do desenvolvimento tecnológico." Tradução livre das autoras.

[14] "Uma medida de alfabetização científica é uma medida de cultura. O currículo tradicional de ciências deixa os estudantes como estrangeiros em sua própria cultura. Um problema essencial que a reforma no ensino de ciências traz é que existem muitos cientistas que são cientificamente analfabetos e muito poucos filósofos, sociólogos e historiadores da ciência e tecnologia que estão interessados nas ciências para a educação básica." Tradução das autoras. P.D. Hurd, Science Literacy: its meaning for american schools. Educational Leadership 16, 13 (1958).

[15] S.A.S. Leite, C.B. Souza, A alfabetização nos cursos de habilitação para o magistério. In: Cadernos de Pesquisa 95, 15 (1995).

[16] Apesar de concordarmos com a utilização da expressão "letramento" ao invés de "alfabetização científica", entendemos que as palavras encerram conceitos por vezes distintos e por isso manteremos neste texto as expressões originalmente utilizadas por seus autores, quando fizermos referências a trabalhos e idéias de outrem. Faremos isso como intuito de não alterarmos o sentido original empregado pelo autor que, na maioria dos casos, trata esse processo de iniciação e de enculturamento como "alfabetização cinetífica" e não como letramento.

[17] Nota dos Editores: Conselho Internacional para a Ciência.

[18] Nota dos Editores: Agência Internacional para a Educação.

[19] Nota dos Editores: Associação Americana para o Avanço da Ciência.

[20] Nota dos Editores: Associação Internacional para a Educação e Tecnologia.

[21] Nota dos Editores: Associação Mundial dos Professores de Ciência.

[22] Nota dos Editores: Conselho Internacional para a Ciência. 
[23] Mito cultural que, ainda que expresso originalmente a partir de uma perspectiva crítica, pode ser reformulado como a utopia que indica o ideal a perseguir. Tradução livre das autoras. M.H. Shamos, The Myth of scientific literacy. New Brunswick: Rutgers University Press (1995).

[24] M. Aktin, J. Helms, Getting seriious about priorities in science education. Studies in Science Education 21, 1 (1993). R.C. Laugksch, Scientific Literacy: A Conceptual Overview. Science Education 84, (1) 71 (2000).

[25] "Não há concenso quanto ao que "alfabetização científica" signifique ou deveria significar.
Ao contrário, todos envolvidos com educação em ciências parecem ter uma noção vaga e fracamente definida do que isso deveria significar." Tradução livre das autoras.

[26] Fazendo alusão aos objetivos modestos que Matthews coloca para a inserção da História, Filosofia e Sociologia (HFS) da Ciência no Ensino de Ciências. Nesse trabalho, o autor argumenta sobre as potencialidades da HFS, mas diz que tem objetivos modestos e foca em apenas alguns aspectos. Aqui, utilizamos a mesma idéia para focalizarmos em quatro aspectos consensuais que o letramento em ciências possui. 